\title{
Relationship between normalized thermal energy and conductivity for cylindrical tokamak geometry
}

\section{Asif}

Department of Physics, COMSATS Institute of Information Technology, Lahore, Pakistan

Received 23 November 2009; revised 4 December 2009; accepted 7 December 2009.

\begin{abstract}
The relation between normalized thermal energy and conductivity, with no particle sources in cylindrical tokamak geometry has been derived. It is obtained, $\beta_{p}=\frac{\sigma_{\perp}}{\sigma_{\|}}$. This relationship is of importance for tokamak confinement and heating.
\end{abstract}

Keywords: Thermal Energy; Cylindrical Geometry; Confinement

\section{INTRODUCTION}

It was previously shown [1], using a numerical analysis, that from the poloidal and toroidal components of Ohm's law for a tokamak, there follows a relation between the normalized thermal-energy content, $\beta$, and the normalized plasma current, $1 / \mathrm{q}$, which only depends on the plasma refueling and not on thermal-energy transport. Previously, the relation was examined only numerically and it is the purpose of [2] to provide an approximate analytical expression for this relation valid for tokamaks, showing explicitly the dependence on the fueling parameters. The relation [3] implies a constraint on the thermal-energy content which cannot be violated, whatever the energy transport present and the plasma heating used. It should be noted that a different relation $[4,5]$ has been derived. Analogous to that of above models, can be obtained by following the same procedure but using Ohm's law [6]. The main focus of this paper is to show the relation between normalized thermal energy and conductivity, with no particle sources in cylindrical tokamak geometry. It is obtained, $\beta_{p}=\frac{\sigma_{\perp}}{\sigma_{\|}}$. This relationship is of importance for tokamak confinement and heating, since it provides a direct constraint on the thermal-energy content which cannot in any case be violated, whatever the energy transport presents.

\section{RELATION BETWEEN NORMALIZED THERMAL ENERGY AND CONDUCTIVITY}

We consider the problem of determining the steady state in cylindrical tokamak geometry with no particle sources. First of all, we recall the expressions for the toroidal and poloidal components of Ohm's law, which can be put in the following form [6], with no particle sources in the limit of large aspect ratio and circular magnetic surfaces:

$$
j_{\phi}=E_{\phi} / \eta_{\text {II }}
$$

$$
\frac{d p}{d r}=-E_{\varphi} B_{\theta} / \eta_{\perp}
$$

where all quantities are surface-averaged and depend on the minor radius $\mathrm{r}, J_{\phi}$ is the toroidal component of the current density, $E_{\phi}$ is the electric field, $\eta_{\mathrm{II}}$ and $\eta_{\perp}$ is the parallel and perpendicular resistivities, $B_{\phi}$ and $B_{\theta}$ are the toroidal and poloidal components of the magnetic field, $\mathrm{p}$ is the plasma pressure. The safety factor is $q=r B_{\phi} / R B_{\theta}$ and $R$ is the major radius. The minor radius of the last closed magnetic surface will be indicated by a, and $B_{\theta}$ and $J_{\phi}$ are related by Ampere's law

$$
j_{\phi}=\frac{1}{\mu_{0} r} \frac{d\left(r B_{\theta}\right)}{d r}
$$

Substituting Eq.1 into Eq.2, one obtains the following equation:

$$
\frac{d p}{d r}=-\frac{\eta_{\mathrm{II}} j_{\phi} B_{\theta}}{\eta_{\perp}}
$$

It should be stressed that, in comparing tokamak experiments with theory, one can certainly use the cylindrical approximation where all quantities depend only on minor radius $r$; however, one must then consider the relations between surface-averaged quantities. In this 
case, the perpendicular component of Ohm's law contains the toroidal corrections (Pfirsch-Schlüter and viscosity or trapping terms) which are proportional to the square of the safety factor and which are absent for the exactly cylindrical case. In this paper these terms were not considered.

The normalized thermal-energy content $\beta_{p}$ can be defined by

$$
\beta_{p}=\frac{2 \mu_{0}}{B_{\theta}^{2}(a)}\langle p\rangle
$$

where $\langle.$.$\rangle indicates the volume average and, in the$ cylindrical approximation,

$$
\langle p\rangle=\frac{2}{a^{2}} \int_{0}^{a} p(r) r d r
$$

So that, if $p(a)=0$, we also have, by integrating by parts,

$$
\langle p\rangle=-\frac{1}{a^{2}} \int_{0}^{a} \frac{d p}{d r} r^{2} d r
$$

Substituting Eq.7 into Eq.5, one obtains the following equation:

$$
\beta_{p}=-\frac{2 \mu_{0}}{a^{2} B_{\theta}^{2}(a)} \int_{0}^{a} \frac{d p}{d r} r^{2} d r
$$

Substituting Eqs.3 and 4 into Eq.8, one obtains the following equation:

$$
\beta_{p}=\frac{\sigma_{\perp}}{\sigma_{\|}}
$$

We have obtained relation between normalized thermal energy (poloidal beta) and conductivity, with no particle sources in cylindrical tokamak geometry. This relationship is a generalization of the usual Bennett relation and it is of importance for tokamak confinement and heating. It should be noted that different relations, analogous to that given above, can be obtained by following the same procedure using Ohm's law [6]. The consideration of the generalized Bennett relation is of importance for the problem of plasma confinement and heating, since it provides a direct constraint on the thermal-energy content which cannot in any case be violated, whatever the energy trans-port present and the heating used.

\section{CONCLUSIONS}

We have obtained relation between normalized thermal energy and conductivity, with no particle sources in cylindrical tokamak geometry. It is obtained, $\beta_{p}=\frac{\sigma_{\perp}}{\sigma_{\|}}$.

This relationship is of importance for tokamak confinement and heating, since it provides a direct constraint on the thermal-energy content which cannot in any case be violated, whatever the energy transport present and the heating used.

\section{REFERENCES}

[1] Segre, S.E. and Zanza, V. (1992) A profile constraint and the value of beta in tokamak experiments. Plasma Physics and Controlled Fusion, 34(11), 1619-1637.

[2] Segre, S.E. and Zanza, V. (1993) A relation between the normalized thermal energy and the safety factor for tokamaks. Nuovo Cimento, 15D(6), 897-901.

[3] Bennett, W.H. (1934) Magnetically self-focusing streams. Physical Review, 45, 890-897.

[4] Asif, M. (2008) Relationship between current density and mass density for ohmic tokamak plasmas. International Journal of Modern Physics B, 22(30), 5329-5333.

[5] Asif, M. (2009) Relationship between viscosity and conductivity for tokamak plasmas. Brazilian Journal of Physics, 39(1), 59-61.

[6] Segre, S.E. and Zanza, V. (1992) Is the perpendicular resistivity in tokamaks really much greater than the Spitzer value? Physics of Fluid, 4(10), 3457-3459. 